\title{
INFRARED EMISSION FROM DUST IN SUPERNOVAE AND SUPERNOVA REMNANTS
}

\author{
ELI DWEK \\ Laboratory for Astronomy and Solar Physics, \\ NASA/ Goddard Space Flight Center
}

\begin{abstract}
The need to replenish the reservoir of interstellar dust grains that are continuously being destroyed in the interstellar medium, and the presence of isotopic anomalies in meteorites, suggest that supernovae may be important sources of interstellar dust. Infrared observations of supernovae or their unmixed ejecta may provide the first direct evidence for newly-formed grains in this environment. The recently discovered supernova, SN 1987A, currently offers the best prospects for observing the actual process of dust formation in a supernova.

In contrast, supernova remnants constitute the most important grain destruction mechanism in the galaxy. Interstellar dust swept up by the expanding blast wave is predominantly heated and destroyed by collisions with a shocked $x$-ray emitting plasma. Infrared observations of remnants can therefore provide valuable information on the interaction between dust particles and a hot gas. This interaction can best be studied in supernova remnants detected with the Infrared Astronomical Satellite (IRAS). This paper reviews what we have learned so far on the subjects of grain formation and destruction by studying the infrared emission from supernovae and supernova remnants.
\end{abstract}

\section{INFRARED EMISSION FROM SUPERNOVA CONDENSATES}

\subsection{SUPERNOVAE AS INTERSTELLAR DUST SOURCES}

To date, the only evidence that dust must form in supernovae (SN) is based on the presence of isotopic anomalies in the meteorites (e.g. Anders, 1989). A weaker argument for the existence of supernova condensates is that they are needed to replenish the amount of interstellar dust that is continuously being destroyed by interstellar shocks (e.g. McKee, 1989). Gehrz (1989) estimates the relative contribution of SN to the interstellar dust population to be negligible compared with that from Red Giant (RG) stars. These estimates are, however, very uncertain, as illustrated in the following example (see also Dwek et al., 1986), where the contribution of SN and RG stars are shown to be comparable. The total mass loss rate from RG stars, integrated over the galaxy, is about $0.3 \mathrm{M}_{\odot} \mathrm{yr}^{-1}$. Adopting a dust-to-gas mass ratio of 0.01 in the flow, we get that the contribution from $R G$ winds to the interstellar dust population is about $3 \times 10^{-3} \mathrm{M}_{\odot} \mathrm{yr}^{-1}$. Consider now the potential contribution of SN. A SN event occurring at a rate of $0.03 \mathrm{yr}^{-1}$ can eject as much 
as $0.1 \mathrm{M}_{\odot}$ of condensable elements to the interstellar medium (ISM). So we see that the contribution of SN may be comparable to that of RG stars. The problem is, however, that we have never actually seen any evidence for dust formation in a supernova - or have we?

\subsection{OBSERVED IR EXCESSES IN SN: ECHOES OR DUST FORMATION?}

Novae exhibit a spectacular behavior in their light curve, presenting unambiguous evidence for the formation of dust in the outflowing nova ejecta. The light curve in many of these objects exhibits a dramatic rise in the infrared (IR), which is concurrent with a rapid drop in the UV-visual (e.g. Gehrz, 1989). Three supernovae: SN 1979C, SN 1980K, and SN 1982E, have exhibited a similar rise in their IR emission without exhibiting a concurrent drop in the UV-visual region of their light curve (which would have provided conclusive evidence for dust formation). The most common explanation for their IR behavior is that the rise in emission is an infrared echo of the initial UV-visual outburst of the supernova caused by a preexisting circumstellar dust shell (Dwek, 1983; Graham and Meikle, 1986).

\subsection{THE INFRARED SPECTRA OF YOUNG, UNMIXED REMNANTS}

Supernova condensates can also be detected in young remnants whose ejecta has not been significantly mixed with the interstellar medium. Cas $\mathbf{A}$, resulting from a SN event that occurred about 300 yrs ago, is a good candidate for such observations. The remnant exhibits a complex structure of fast-moving optical nebulosities whose composition suggests that they represent undiluted material that has been ejected from the interior of the exploded star (Chevalier and Kirshner, 1979). In addition to these fast-moving knots, there may be an unobserved component of slow-moving SN ejecta. X-ray observations of the remnant (Murray et al., 1979) suggest that in addition to the expanding SN blast wave there is a reverse shock, propagating inwards through the SN cavity. This reverse shock can collisionally heat any supernova condensates that may be present in the SN ejecta. Dwek et al. (1987) examined the possibility that the IRAS observations of the remnant may be interpreted in terms of IR emission from these dust particles. The results show that the IR spectrum can indeed be thermal emission from dust heated by the reverse shock. This interpretation is, however, not unique: the IR spectrum can equally well, if not better, be fitted by a model in which the emission originates from interstellar/circumstellar dust grains, heated by the outwardly expanding SN blast wave.

\subsection{IS DUST FORMING IN SN 1987A?}

Since this talk was given in July 1988, there has been an exciting new development in the IR spectrum of SN $1987 \mathrm{~A}$. A recent observation of the supernova showed that the 8-13 $\mu \mathrm{m}$ continuum emission, which has been fading over the past year, brightened on July 24,1988 , by almost a factor of two since the last observations taken on June 1 (Smith, James and Aitken, 1988). If, as pointed out by Smith et al. (1988), there is no simultaneous increase at the shorter IR wavelengths, then this spectral evolution will strongly suggest the appearance of thermal emission from dust in the supernova spectrum. If so, what is the origin of the emitting dust? Are we witnessing the onset of dust formation in the supernova ejecta, or are we 'merely' 
seeing the delayed IR emission from pre-existing dust in the circumstellar shell that has been detected from UV observations (e.g. Kirshner, 1988) of the supernova?

The fact that the 8-10 $\mu \mathrm{m}$ flux actually increased, argues against it being an echo, since echo models (e.g. Dwek, 1983) predict the existence of a phase of constant IR emission lasting for a period of $2 R / c$, where $R\left(\approx 1 \times 10^{18} \mathrm{~cm}\right.$ from UV observations of the SN) is the shell radius, and $c$ the speed of light. However, asymmetries in the distribution of dust in the shell can always be invoked to explain the observed changes in the IR emission with an echo model.

The composition of the emitting dust may provide an important clue to its origin. Circumstellar dust is expected to be composed of silicate-type material since preSN models (e.g. Woosley, 1988) suggest that the circumstellar shell is oxygen rich. Its composition can then be inferred from its 9.7 or $18 \mu \mathrm{m}$ emission features. SN condensed dust may have a different composition depending on that of the dust forming layer. Depending on the degree of mixing, we may expect sulfates, carbonor silicate-type dust to form in the $\mathrm{SN}$ ejecta.

The formation of dust in the SN ejecta will result in the depletion of various refractory elements from the gas phase. If the dust is forming in the same region that gives rise to the various IR lines of $\mathrm{Fe}, \mathrm{Co}, \mathrm{S}, \mathrm{Ni}, \mathrm{Ar}$, and $\mathrm{Ne}$ (Rank et al., 1988; Witteborn et al., 1988; Aitken et al., 1988; and Moseley et al., 1988), then we would be able to actually observe the precipitation of the various refractory elements from the gas phase.

Another indication that the rise in the IR is due to dust formation will be a concomitant decrease in the UV-visual light curve in excess of that expected due to the increased transparency of the ejecta. Simultaneous observations of the SN at $\gamma, \mathrm{x}$-ray, UV, optical, and IR wavelengths are important to ascertain this point.

Finally, dust, forming in the ejecta, will be heated by the UV-visual energy available at the time of its formation. Accordingly, there are tight energy constraints on the possible IR intensity. Such constraints do not apply to the echo model, in which the emitting dust has been heated by the UV-visual output of the SN during the first 2-4 months after the outburst. The origin of the emitting dust may therefore be established by the long-time behavior of the UV-optical and IR spectrum of the supernova.

\section{INFRARED EMISSION FROM SUPERNOVA REMNANTS}

IRAS observations of supernova remnants (SNRs) provided astronomers with the first observational evidence of shock-heated dust in the interstellar medium (ISM). A review of the early searches for infrared (IR) emission from SNRs and analyses of remnants detected with IRAS were presented by Dwek (1988). Several additional remnants have been studied in detail since that review: CTB 80 (Fesen, Shull, and Saken, 1988; hereafter FSS), OA 184 (Leahy and Marshall (1988), and Puppis A (Arendt et al., 1988; Arendt, Dwek, and Petre, 1988; hereafter ADP). In this section I will emphasize IR studies of remnant morphology, and IR manifestations of basic interactions (heating, sputtering) between dust particles and a hot plasma. 


\subsection{THE IR MORPHOLOGY OF SNRS AND THEIR AMBIENT ISM}

The IR images of SNRs provide an excellent view of the morphology of the remnants and the medium into which they are expanding, and of the interaction between the two. Analysis of the Cygnus Loop (Braun, 1986b), for example, confirmed that the remnant is expanding into a preexisting cavity created by stellar mass loss during the presupernova phase of the progenitor star's evolution. The IR observations of IC 443 (Braun 1986a; Mufson et al., 1986) show that the IR morphology of the remnant is dominated by its interaction with a molecular cloud. Puppis A (Arendt et al., 1989; ADP) and RCW 86 (Dwek and Spiesman, 1988) are expanding into media with strong density gradients, offering a natural explanation for the strongly asymmetric x-ray appearance of these remnants (Petre et al., 1982; Pisarski, Helfand, and Kahn, 1984). IRAS images of CTB 80 (FSS) revealed an IR emission shell, presumably associated with the pulsar. According to FSS, the interaction of the pulsar with this shell's magnetic field can explain the unusual radio structure of the remnant.

To date, IR images of SNRs have provided no evidence of supernovae expanding into a low-density interstellar medium containing isolated cloudlets, as envisioned by McKee and Ostriker (1978). The x-ray peak in the map of Puppis A (region C in Szymkowiak, 1985), originally thought to be an isolated cloudlet, was considered as supporting evidence for that picture (Petre et al., 1982). The IR observations revealed that the $\mathrm{x}$-ray peak has an associated IR maximum, and that it constitutes part of a larger molecular complex abutting the northeast section of the remnant (Arendt et al., 1988; ADP). The picture revealed by the IRAS data is more consistent with SNRs expanding in an inhomogeneous ISM with densities $>0.1 \mathrm{~cm}^{-3}$ and structures comparable to the size of the remnant. However, it should be emphasized that SNRs expanding into media with densities lower than $\sim 0.1 \mathrm{~cm}^{-3}$ are probably too faint to have been observed with the IRAS above the intervening cirrus and other sources of IR emission.

\subsection{DUST TEMPERATURE IN A HOT GAS}

A recent analysis of the interaction between dust particles and a hot plasma was presented by Dwek (1987). I will emphasize here two important results of that analysis, relevant to the following discussion: the dependence of dust temperature on plasma conditions, and its dependence on grain size.

Figure 1 (from Dwek, 1987) depicts the temperature, $T_{d}$, of $0.1 \mu \mathrm{m}$ silicate and graphite grains that are collisionally heated by a plasma with an electron density of $1 \mathrm{~cm}^{-3}$, as a function of electron temperature $T$. The dust temperature initially rises with $T$ as all the energy of the impinging electrons is deposited in the dust. Above a gas temperature of $\approx 2 \times 10^{7} \mathrm{~K}$, the electrons penetrate through the grains and $T_{d}$ reaches a plateau value of $\approx 58 \mathrm{~K}$. Higher grain temperatures can only be attained by increasing the ambient gas density. This behavior of $T_{d}$ versus $T$ suggests that in any IR analysis we should divide all remnants into two categories: young remnants, defined here as remnants in which the postshock temperature is $\geq 2 \times 10^{7} \mathrm{~K}$ (or expansion velocities above $\sim 1200 \mathrm{~km} \mathrm{~s}^{-1}$ ); and older remnants, characterized by postshock temperatures $\leq 2 \times 10^{7} \mathrm{~K}$ (or expansion velocities below $\sim 1200 \mathrm{~km}$ $\mathbf{s}^{-1}$ ). In young remnants $T_{d}$ is essentially independent of the gas temperature, and their IR spectra are excellent diagnostics of gas density. In older remnants the 


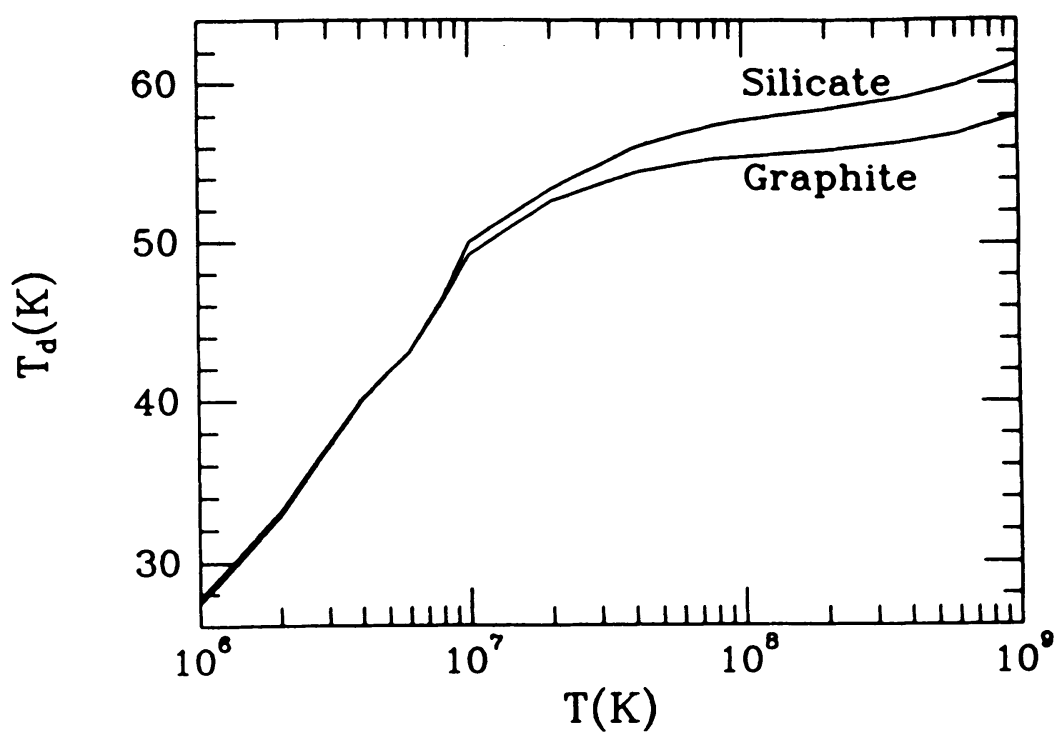

Fig. 1. The temperature of $0.1 \mu \mathrm{m}$ silicate and graphite dust particles is depicted as a function of gas temperature $T$, for a gas density of $n=1 \mathrm{~cm}^{-3}$.

dust temperature constrains the allowable combinations of plasma densities and temperatures.

The same effect of increasing grain transparency with electron energy also determines the functional behavior of grain temperature with size. Figure 2 (taken from Dwek, 1987) shows that at electron temperatures above $\approx 2 \times 10^{7} \mathrm{~K}$, the dust temperature is essentially independent of grain sizes up to $\approx 0.2 \mu \mathrm{m}$. This result is due to the fact that the dust heating efficiency by electronic collisions and its infrared absorption coefficient have a similar functional dependence on grain size.

So far, I have assumed that the dust temperature can be characterized by its equilibrium value, obtained by balancing the dust heating rate by particle collisions to its cooling rate by IR emission. However, below a given grain size, (approximately $200 \AA$ in most remnants), the temperature of a dust particle will fluctuate with time. This effect is a natural consequence of the heat capacity and cross section of these small dust particles: individual electron hits raise the grain temperature well above its equilibrium value, and the grain cooling time at these temperatures is significantly faster than the time between electronic collisions. 


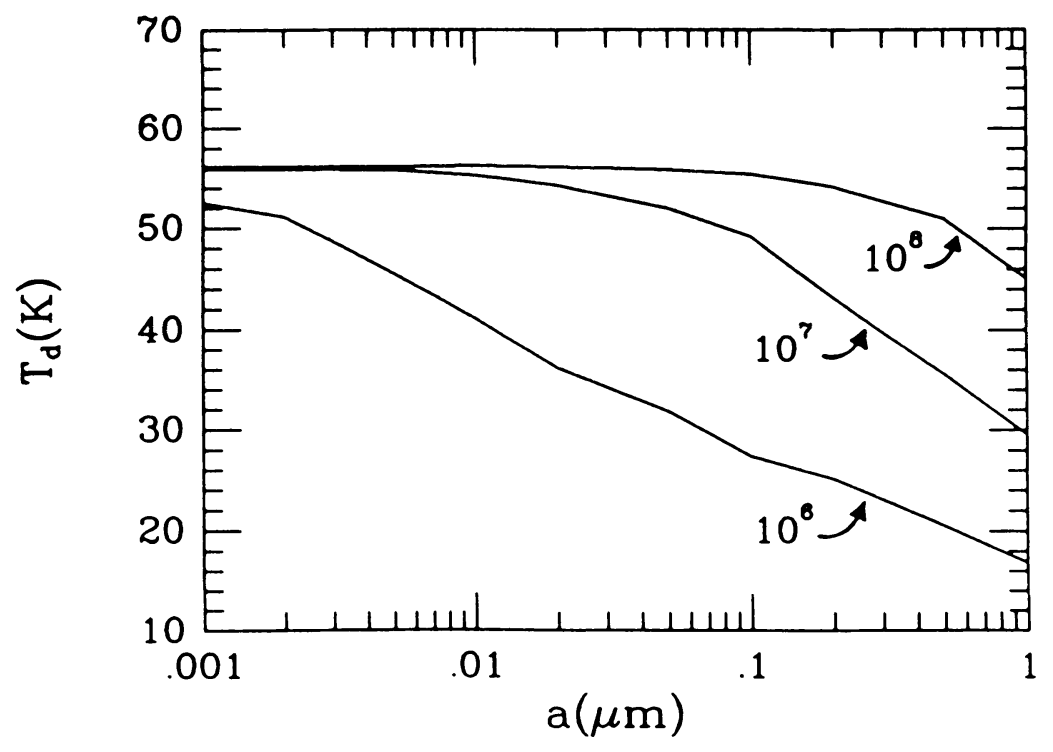

Fig. 2. The variation of the temperature of a graphite grain is depicted here as a function of grain size for $\mathrm{n}=1 \mathrm{~cm}^{-3}$ and various values of the gas temperature $T(K)$.

\subsection{EVIDENCE FOR VERY SMALL DUST PARTICLES IN A SHOCKED GAS}

The results described above suggest that in young supernova remnants such as CasA, Tycho, and Kepler, characterized by gas temperatures in excess of $2 \times 10^{7}$ $K$, the infrared spectrum should be well described by a single-temperature dust model. Yet, the observed IR spectra of these remnants (Braun, 1987; Dwek et al., 1987) show a significant excess of $12 \mu \mathrm{m}$ emission above that expected from dust particles radiating at the equilibrium temperature. Simply extending the grain size distribution to very small particles $(a \approx 3 \AA)$ will not cause a broadening in the IR spectrum, since the equilibrium dust temperature is essentially constant with grain size. The $12 \mu \mathrm{m}$ flux from these remnants can only be explained if the temperature of these dust particles fluctuates, reaching values of $\sim 300 \mathrm{~K}$ for brief intervals of time. The observed $12 \mu \mathrm{m}$ excess in these remnants therefore provides solid proof for the existence of dust particles with sizes between $\sim 10-200 \AA$ in the remnant, and for the stochastic nature of their heating mechanism. Furthermore, the lifetime of these remnants is short ( $<400 \mathrm{yrs}$ ), so these very small particles could not have been created by the destruction of larger $(a \approx 250 \AA)$ particles in the shocked gas (see below). This suggests that the grain size distribution in the pre-shocked gas must have contained the very small dust particles needed to explain the excess 12 $\mu \mathrm{m}$ emission in the spectrum of these remnants. 


\subsection{EVIDENCE FOR GRAIN DESTRUCTION}

In a hot gas, dust particles are destroyed by sputtering on a timescale given by $t(\mathrm{yr}) \approx 10^{6} \mathrm{a}(\mu \mathrm{m}) / n$, where $n$ is the gas density in $\mathrm{cm}^{-3}$ and $a$ is the radius in microns (Draine and Salpeter, 1979; Seab, 1987). The lifetime of a $0.1 \mu \mathrm{m}$ dust particle embedded in a $10 \mathrm{~cm}^{-3}$ gas is $\approx 10^{4} \mathrm{yrs}$, longer than the shock crossing time of all bright $\mathrm{x}$-ray remnants observed with the IRAS. It should therefore not be surprising to find dust particles in SNRs, a fact clearly demonstrated by the IRAS observations. Sputtering, will, however, reduce the relative number of small dust particles in the size distribution. This effect will suppress the short wavelength IR emission from the dusty plasma.

To demonstrate that dust destruction is taking place in the remnant, one must assume knowledge of the initial grain size distribution. In Cas A (Dwek et al., 1987) and Puppis A (Arendt et al., 1988; ADP), the initial grain size distribution was taken to be a Mathis, Rumpl, and Nordsieck (1977) distribution extended to very small grain sizes of $\sim 3 \AA$ (hereafter referred to as an EMRN distribution). In Cas A the EMRN distribution produced an IR flux that was clearly in excess of the observed $12 \mu \mathrm{m}$ IR emission. In region C of Puppis A (see Figure 3 in ADP) the EMRN grain size distribution produced a flux that, compared with the observations, was too high at $25 \mu \mathrm{m}$, and too low at $60 \mu \mathrm{m}$. An improved fit to the observed IR fluxes in Cas A and region C in Puppis A was obtained after the grain size distribution was modified by sputtering. The amount of sputtering needed to improve the fit to the observed fluxes determines the residence time of the dust in the hot gas, or the time since the gas was swept up by the expanding SN blast wave. For regionC in Puppis A, ADP found that the grain residence time in the shocked gas is $300 \pm 150$ yrs.

The examples above show that detailed analysis of the IR emission from various regions in extended SNRs can, in principle, be used to reconstruct the evolutionary history of the remnant. In practice, such a task is complicated by the uncertainties in the short wavelength IR emission, and in the details of the initial grain size distribution.

ACKNOWLEDGEMENTS. This research was partially supported under NASA's IRAS Data Analysis Program.

\section{REFERENCES}

Aitken, D. K., Smith, C. H., James, S. D., Roche, P. F., Hyland, A. R., and McGregor, P. J. 1988, M. N.R.A.S. 281,7 .

Anders, E. 1989, in IAU Symposium 195, Interstellar Dust, eds. L. J. Allamandola and A. G. G. M. Tielens, (Dordrecht: Kluwer), p. 389

Arendt, R., Dwek, E., and Petre, R. 1989, in Interstellar Dust Contributed Papers, eds. A. G. G. M. Tielens and L. J. Allamandola, NASA CP-3036.

Arendt, R., Dwek, E., Petre, R., and Dickel, J. R. 1988, in preparation.

Braun, R. 1986a, Astr. Ap., 164, 193.

-. 1986b, Astr. Ap., 164, 208.

- 1987, Astr. Ap., 171, 233.

Chevalier, R. A., and Kirshner, R. P. 1979, Ap. J., 283, 154. 
Draine, B. T., and Salpeter, E. E. 1979, Ap. J., $231,77$.

Dwek, E. 1983, Ap. J., 274, 175.

Dwek, E. et al.1986, in Interrelationships Among Circumstellar, Interstellar, and Interplanetary Dust, eds. J. A. Nuth, III, and R. E. Stencel, NASA CP-2403, p. WG 1.

Dwek, E. 1987, Ap. J., 322, 812.

Dwek, E., Dinerstein, H. L., Gillett, F. C., Hauser, M. G., and Rice, W. L. 1987, Ap. J., 315, 571.

Dwek, E. 1988, in Supernova Remnants and the Interstellar Medium, IAU Colloquium 101, eds. R. S. Roger and T. L. Landecker, (Cambridge: Cambridge Univ. Press), p. 363.

Dwek, E., and Spiesman, W. J. 1988, in preparation.

Fesen, R. A., Shull, J. M., and Saken, J. M. 1988, Nature, in press (FSS).

Gehrz, R. D. 1989, in IAU Symposium 195, Interstellar Dust, eds. L. J. Allamandola and A. G. G. M. Tielens, (Dordrecht: Kluwer), p. 445.

Graham, J. R., and Meikle, W. P. S. 1986, M. N. R. A. S., 221, 789.

Kirshner, R. P. 1988, in Supernova 1987A in the Large Magellanic Cloud, eds. M. Kafatos and A. G. Michalitsianos, (Cambridge: Cambridge Univ. Press), p. 87.

Leahy, D. A., and Marshall, C. R. 1988, M. N.R. A. S., in press.

Mathis, J. S., Rumpl, W., and Nordsieck, K. H. 1977, Ap. J., 217, 425.

McKee, C. F. 1989, in IAU Symposium 195, Interstellar Dust, eds. L. J. Allamandola and A. G. G. M. Tielens, (Dordrecht: Kluwer), p. 431.

McKee, C. F., and Ostriker, J. P. 1977, Ap. J., 218, 148.

Murray, S. S., Fabbiano, G., Fabian, A. C., Epstein, A., and Giacconi, R. 1979, Ap. J. (Letters), 284, L69.

Moseley, S. H., Dwek, E., Silverberg, R. F., Glaccum, W. J., Graham, J. R., and Loewenstein, R. F. 1988, Ap. J., submitted.

Mufson, S. L., McCollough, M. L., Dickel, J. R., Petre, R., White, R., and Chevalier, R. 1986, A. J., $92,1349$.

Petre, R., Canizares, C. R., Kriss, G. A., and Winkler, P. F. 1982, Ap. J., 258, 22.

Pisarski, R. L., Helfand, D. J., and Kahn, S. M. 1984, Ap. J., 277, 710.

Rank, D. M., Pinto, P. A., Woosley, S. E., Bregman, J. D., Witteborn, F. C., Axelrod, T. S., and Cohen, M. 1988, Nature, 391, 505.

Seab, C. G. 1987, in Interstellar Processes, eds. D. Hollenbach, and H. A. Thronson, Jr., (Dordrecht: Reidel), p. 491.

Smith, C., James, S., and Aitken, D. 1988, IAU Circ. No. 4645.

Szymkowiak, A. E. 1985, Ph. D. Thesis, NASA TM 86169.

Witteborn, F., Bregman, J., and Wooden, D. 1988, IAU Circ. No. 4592.

Woosley, S. E. 1988, Ap. J., 330, 218. 